\title{
STATISTICAL STABILITY FOR DETERMINISTIC AND RANDOM DYNAMICAL SYSTEMS
}

\author{
HARRY CRIMMINS
}

(Received 12 July 2021; first published online 30 September 2021)

2020 Mathematics subject classification: primary 37C30; secondary 37C75, 37H30, 37M25.

Keywords and phrases: hyperbolic dynamical system, random dynamical system, stability, spectral perturbation.

It is well known that sufficiently smooth, hyperbolic dynamical systems admit strong statistical descriptions; for example, limit laws such as a central limit theorem or a large-deviation principle. Given the existence of these laws one is led to the question of their stability: do nearby systems have similar statistical descriptions and to what extent can one numerically approximate the statistics of any particular system? In this thesis such questions are investigated by building on the so-called functional analytic approach and in particular the spectral perturbation theory of Keller and Liverani. For deterministic systems it is shown that the Keller-Liverani perturbation theory is compatible with the naive Nagaev-Guivarc'h method, the method used to obtain the aforementioned statistical limit laws, yielding a general framework for deducing the statistical stability of deterministic dynamical systems under a variety of perturbations. This theory is then applied to piecewise-expanding maps in one and many dimensions, in addition to Anosov maps on tori. Of particular note is the development of new, efficient and rigorous numerical methods for the approximation of the statistical properties of multidimensional piecewise-expanding maps and Anosov maps. In the second part of the thesis this programme is begun again for random systems. Here there is no analogue of the Keller-Liverani perturbation theory and so an appropriate random version of the theory is developed. This theory is then applied to smooth random expanding maps on the circle, and the stability of some basic statistical properties is deduced with respect to fibre-wise deterministic perturbations and a Fourier-analytic numerical method.

Some of this research has been published in [1-3].

Thesis submitted to the University of New South Wales in September 2020; degree approved on 23 February 2021; supervisor Gary Froyland.

(C) 2021 Australian Mathematical Publishing Association Inc. 


\section{References}

[1] H. Crimmins, 'Stability of hyperbolic Oseledets splittings for quasi-compact operator cocycles, Preprint, 2019, arXiv:1912.03008.

[2] H. Crimmins and G. Froyland, 'Stability and approximation of statistical limit laws for multidimensional piecewise expanding maps', Ann. Henri Poincaré 20 (2019), 3113-3161.

[3] H. Crimmins and G. Froyland, 'Fourier approximation of the statistical properties of Anosov maps on tori', Nonlinearity 33 (2020), Article no. 6244.

HARRY CRIMMINS, School of Mathematics and Statistics,

University of New South Wales, Sydney, NSW 2052, Australia

e-mail: harry.crimmins@unsw.edu.au 